\title{
Tangential vitreous traction: a possible mechanism of development of cystoid macular edema in retinitis pigmentosa
}

This article was published in the following Dove Press journal:

Clinical Ophthalmology

2I February 201 I

Number of times this article has been viewed

\author{
Mikiko Takezawa \\ Soichi Tetsuka \\ Akihiro Kakehashi \\ Department of Ophthalmology, Jichi \\ Medical University, Saitama Medical \\ Center, Saitama, Saitama, Japan
}

\begin{abstract}
We report the possible mechanism of development of cystoid macular edema (CME) in retinitis pigmentosa (RP) in the case of a 68-year-old woman with RP and CME in the right eye and resolving CME in the left eye. Spectral domain optical coherence tomography showed $\mathrm{CME}$ and posterior vitreoschisis in the nasal quadrant of the fundus without a posterior vitreous detachment (PVD). This vitreous pathology suggested bilateral thickening and shrinkage of the posterior vitreous cortex. In the right eye, CME was evident with no vitreofoveal separation. However, in the left eye, minimal change was seen in the CME associated with a focal shallow PVD over the fovea. The best-corrected visual acuity (BCVA) in the left eye increased to 0.3 from 0.157 years after the first visit. Tangential vitreous traction on the macula may have caused the CME in the right eye. The shallow PVD over the fovea might have released the tangential vitreous traction from the fovea, induced spontaneous resolution of the CME, and improved the BCVA in the left eye.
\end{abstract}

Keywords: retinitis pigmentosa, cystoid macular edema, posterior vitreous detachment, posterior vitreoschisis, optical coherence tomography

\section{Introduction}

Cystoid macular edema (CME) is sometimes complicated by retinitis pigmentosa (RP) and may worsen visual acuity (VA) at a later stage. ${ }^{1,2}$ The prevalence of CME in patients with RP has been reported to be about $20 \%$ based on fluorescein angiography (FA) and fundus examination. ${ }^{3,4}$ Recent studies using time domain optical coherence tomography (OCT) have reported that OCT is more sensitive for detecting CME and atrophy in patients with RP than conventional ocular examinations, including FA and ophthalmoscopy. ${ }^{5,6}$ However, the pathogenesis of the development of CME in RP has not been clarified. CME develops in RP bilaterally and sometimes unilaterally. However, advanced atrophic macular changes were found more frequently than CME in RP. ${ }^{4}$ Fishman et al speculated that macular cysts resulted from changes in the vitreomacular interface. ${ }^{3}$ The most recent generation of OCT, spectral domain OCT (SD-OCT), may detect the detailed changes in the vitreoretinal relationship. In the current report, we postulated a possible mechanism of the development of CME in a patient with RP by evaluating the vitreoretinal relationship using SD-OCT.

\section{Case report}

A 68-year-old woman was referred to us for evaluation of her RP. Typical fundus changes of the bone spicule intraretinal pigmentation in the mid-periphery and attenuated retinal vessels (Figure 1) and almost extinguished electroretinogram (ERG) 


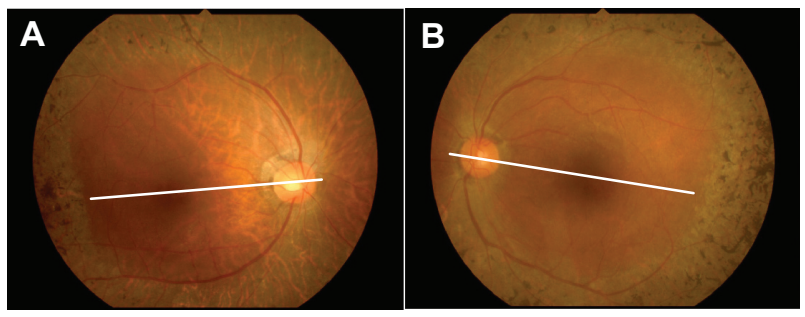

Figure I Fundus appearance of the right A) and left B) eyes. The bone spicule deposits are clustered just anterior to the posterior pole. The cystoid macular edema is unremarkable in this photograph of both eyes. The white line indicates the optical coherence tomography scan line in Figure 4.

recordings of all responses by portable ERG (LE-3000 ${ }^{\circledR}$; Tomey Corporation, Nagoya, Japan), including single flash, cone, rod, and flicker (Figure 2), strongly supported the previous diagnosis of RP. Goldmann kinetic perimetry showed extensive bilateral visual field loss (Figure 3). Other family members had normal vision. The patient did not have a history of diabetes, uveitis, or ocular surgery. Her initial best-corrected visual acuity (BCVA) was 0.5 with $+0.25-0.5$ axis $75^{\circ}$ in the right eye and 0.15 with $+2.0-1.5$ axis $110^{\circ}$ in the left eye. Slit-lamp biomicroscopy showed no evidence of a posterior vitreous detachment (PVD) or spindle-shaped vitreous opacities bilaterally. Seven years after the first visit, SD-OCT (Cirrus ${ }^{\mathrm{TM}}$ HD OCT; Carl Zeiss Meditec, Dublin, CA) (Figure 4) showed CME and no evidence of PVD but posterior vitreoschisis in the nasal quadrant bilaterally. In the right eye (Figure 4A), CME was evident, and no vitreofoveal separation was observed. However, in the left eye (Figure 4B), minimal changes were seen in the $\mathrm{CME}$ associated with a focal shallow PVD over the fovea. The BCVA of her right eye was unchanged at 0.5 , but the BCVA of her left eye improved to 0.3 from 0.15 at the first visit.

\section{Discussion}

The pathogenesis of CME associated with RP is uncertain. Some reports have suggested that retinal edema may be caused by inflammation resulting from degeneration of the outer retina. ${ }^{7,8}$ Other reports have suggested that some
Normal case
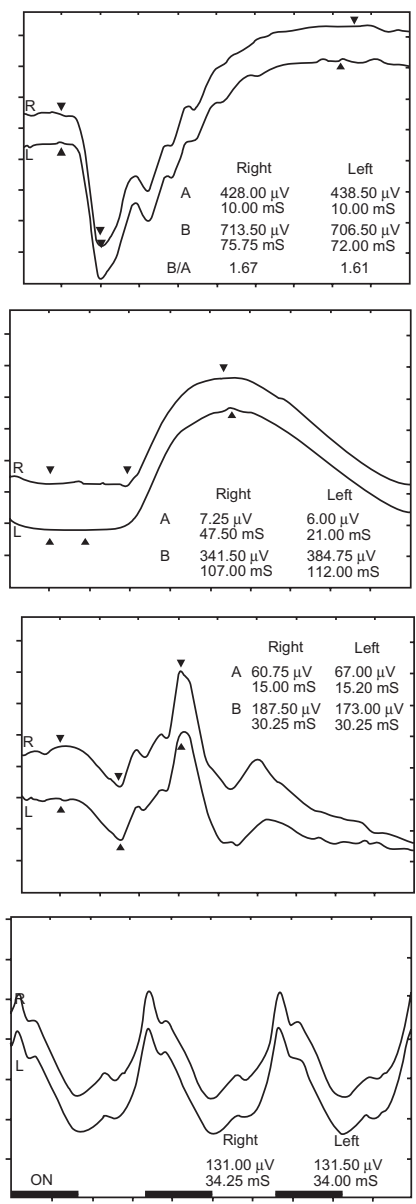

Flash ERG

Current case
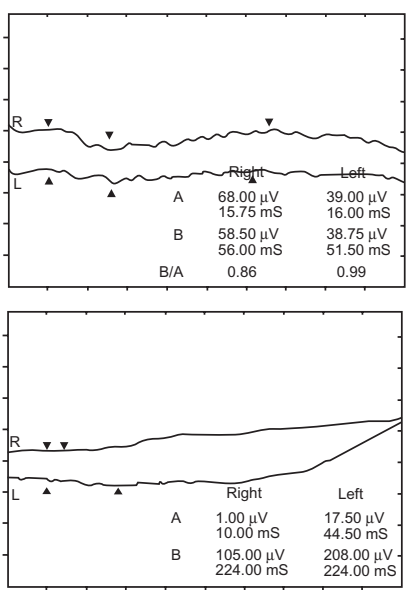

Cone response

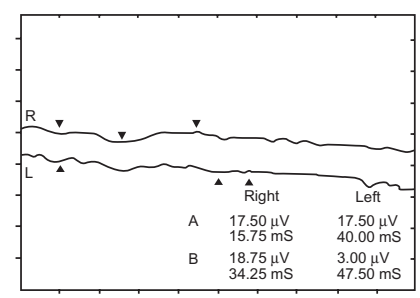

$30 \mathrm{~Hz}$ flicker

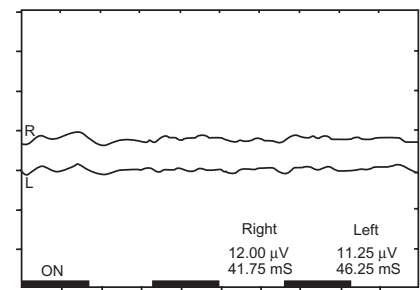

Figure 2 The electroretinogram (ERG) of the current case compared with that of a normal eye. The patient has no ERG responses, including flash, cone, rod, and 30-Hz flicker. 


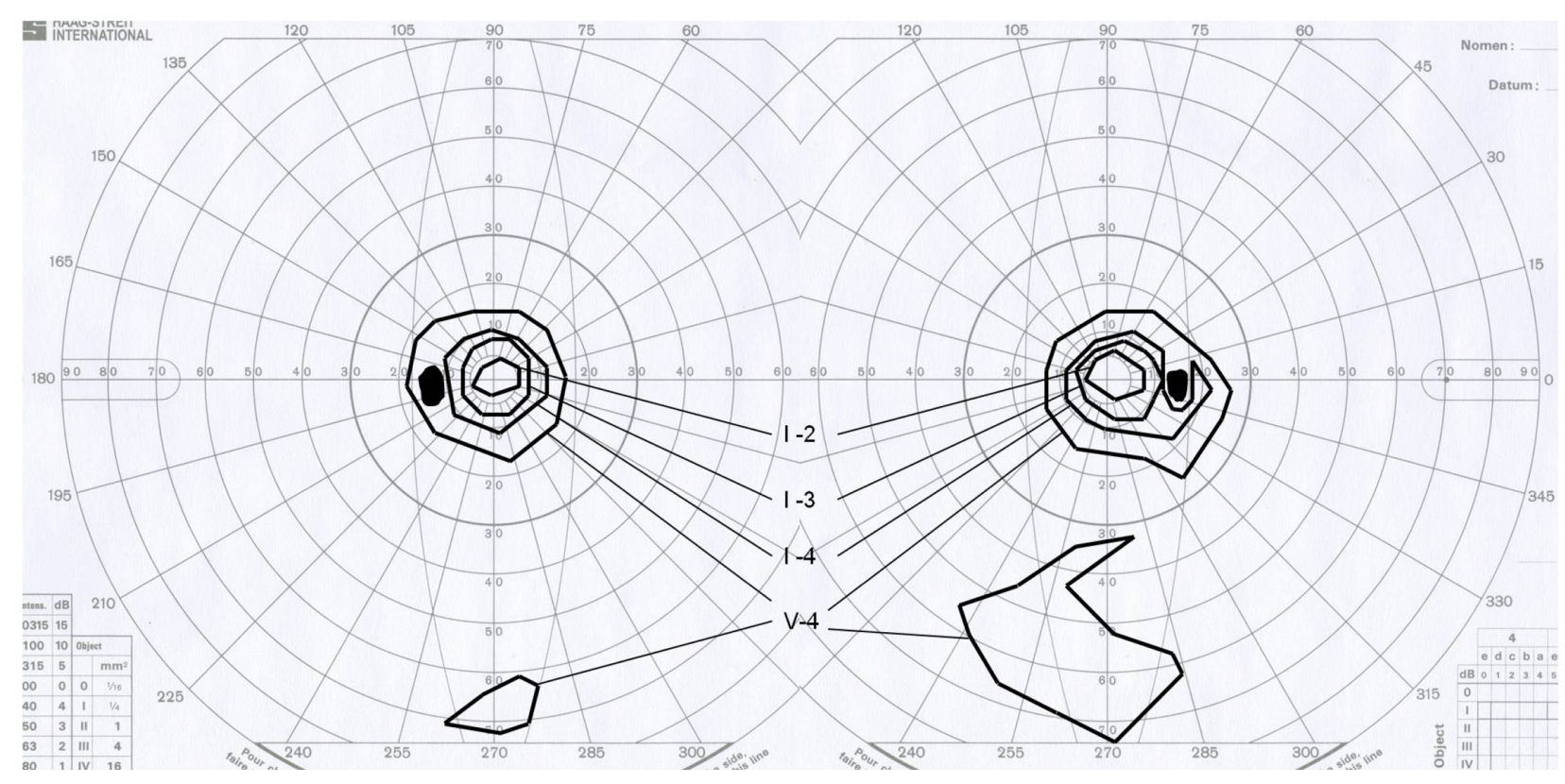

Figure 3 Goldmann kinetic perimetry indicates the marked constriction of the V-4 isopter and the inferior peripheral remaining visual field.

changes in the vitreomacular interface may cause the macular pathology in patients with $\mathrm{RP}^{3}{ }^{3}$ and based on that hypothesis vitrectomy was performed to treat the CME. ${ }^{6}$ CME can develop bilaterally and unilaterally. However, atrophic macular changes are seen frequently in RP. There seems to be no relationship between the development of $\mathrm{CME}$ and the RP stage or inheritance type. Although other coexisting factors such as diabetes, uveitis, or ophthalmic surgery may affect the pathogenesis of CME, it can develop in patients with RP without such risk factors. Therefore, focal deterioration must affect the macula for CME to develop in RP. The current patient had no evidence of PVD bilaterally

A
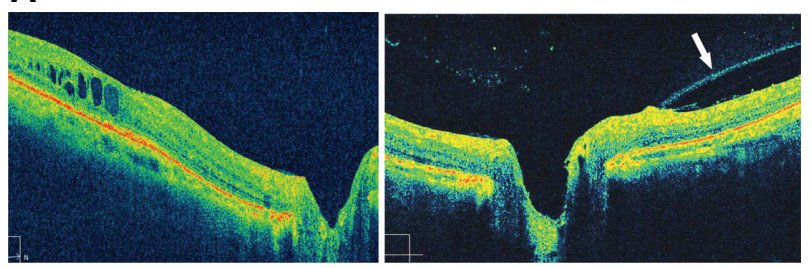

B

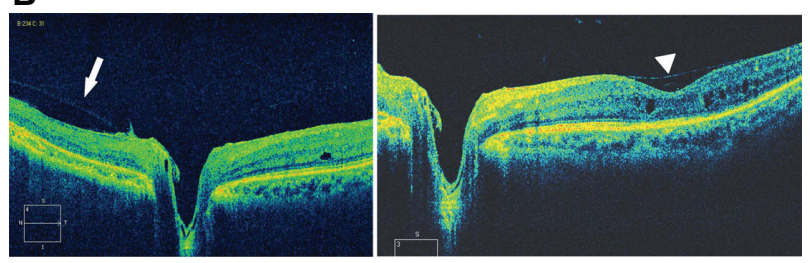

Figure 4 Spectral domain optical coherence tomography images of the right $\mathbf{A}$ ) and left B) eyes. In the right eye, cystoid macular edema (CME) at the fovea and posterior vitreoschisis (arrow) in the nasal quadrant is seen without a posterior vitreous detachment (PVD). In the left eye, minimal CME at the fovea with a focal PVD (arrowhead) and posterior vitreoschisis (arrow) in the nasal quadrant are seen. by biomicroscopy. SD-OCT also showed bilateral posterior vitreoschisis in the nasal quadrant of the fundus without a PVD. Posterior vitreoschisis is seen frequently in retinal vascular diseases and results from strong vitreous adhesion to the retina and shrinkage of the vitreous. ${ }^{9}$ Bilateral CME was observed by SD-OCT, but the CME changes were minimal in the left eye. SD-OCT also showed a shallow focal PVD just over the fovea in the left eye. The BCVA improved to 0.3 from 0.15 at the first visit. The shallow PVD over the fovea might have induced spontaneous resolution of CME and the improved BCVA in the left eye. In other words, strong tangential vitreous traction is a possible mechanism of the development of CME in the right eye.

Vitreous abnormalities have been well documented in all RP stages. In early-stage RP, dust-like particles were seen in the clear and solid vitreous. In the advanced stage, an atypical PVD progressed with formation of condensed irregular fiber aggregates. ${ }^{10}$ The morphologic vitreous changes in the current case, ie, no PVD without significant vitreous opacity, did not correspond well to the vitreous findings reported previously. However, posterior vitreoschisis may be the stage before the advanced stage that shows atypical PVD with formation of condensed irregular fiber aggregates. The atypical PVD found in the advanced stage may not be a true PVD but could be an advanced type of posterior vitreoschisis.

The current case suggested a new effective treatment option for CME in RP vitrectomy combined with internal limiting membrane (ILM) removal. Cases with RP have strong vitreous adhesion to the retina; therefore, simple vitrectomy 
may be ineffective for releasing the tangential vitreous traction on the fovea. Numerous reports have discussed medical and surgical treatments. Some medical treatments, such as oral carbonic anhydrase inhibitor acetazolamide, ${ }^{11-13}$ intravitreous triamcinolone, ${ }^{14}$ and antivascular endothelial growth factor agents, ${ }^{15}$ have been reported; however, most seem to only temporarily affect the CME. However, some studies have reported successful results with vitrectomy combined with ILM removal in some RP cases with CME. ${ }^{6,16}$ The current case supports vitrectomy for treating CME in RP. However, cases with a shallow PVD over the fovea, even with extensive CME, should not undergo surgery, because there is a chance that the VA can recover in the future.

\section{Disclosure}

The authors report no conflicts of interest in this work.

\section{References}

1. Fetkenhour CL, Choromokos E, Weinstein J, Shoch D. Cystoid macular edema in retinitis pigmentosa. Trans Sect Ophthalmol Am Acad Ophthalmol Otolaryngol. 1977;83(3 Pt 1):OP515-OP521.

2. Newsome DA. Retinal fluorescein leakage in retinitis pigmentosa. $A m$ J Ophthalmol. 1986;101(3):354-360.

3. Fishman GA, Fishman M, Maggiano J. Macular lesions associated with retinitis pigmentosa. Arch Ophthalmol. 1977;95(5):798-803.

4. Fishman GA, Maggiano JM, Fishman M. Foveal lesions seen in retinitis pigmentosa. Arch Ophthalmol. 1977;95(11):1993-1996.
5. Hirakawa H, Iijima H, Gohdo T, Tsukahara S. Optical coherence tomography of cystoid macular edema associated with retinitis pigmentosa. Am J Ophthalmol. 1999;128(2):185-191.

6. Hagiwara A, Yamamoto S, Ogata K, et al. Macular abnormalities in patients with retinitis pigmentosa: prevalence on OCT examination and outcomes of vitreoretinal surgery. Acta Ophthalmol. March 8, 2010. [Epub ahead of print]

7. Spalton DJ, Bird AC, Cleary PE. Retinitis pigmentosa and retinal oedema. Br J Ophthalmol. 1978;62(3):174-182.

8. Uliss AE, Gregor ZJ, Bird AC. Retinitis pigmentosa and retinal neovascularization. Ophthalmology. 1986;93(12):1599-1603.

9. Kakehashi A, Schepens CL, de Sousa-Neto A, et al. Biomicroscopic findings of posterior vitreoschisis. Ophthalmic Surg. 1993;24(12): 846-850.

10. Pruett RC, Albert DM. Vitreous degeneration in myopia and retinitis pigmentosa. In: Schepens CL, Neetens A, editors. The Vitreous and Vitreoretinal Interface. New York: Springer-Verlag; 1987.

11. Cox SN, Hay E, Bird AC. Treatment of chronic macular edema with acetazolamide. Arch Ophthalmol. 1988;106(9):1190-1195.

12. Chen JC, Fitzke FW, Bird AC. Long-term effect of acetazolamide in a patient with retinitis pigmentosa. Invest Ophthalmol Vis Sci. 1990; 31(9): 1914-1918.

13. Orzalesi N, Pierrottet C, Porta A, Aschero M. Long-term treatment of retinitis pigmentosa with acetazolamide. A pilot study. Graefes Arch Clin Exp Ophthalmol. 1993;231(5):254-256.

14. Saraiva VS, Sallum JM, Farah ME. Treatment of cystoid macular edema related to retinitis pigmentosa with intravitreal triamcinolone acetonide. Ophthalmic Surg Lasers Imaging. 2003;34(5):398-400.

15. Artunay O, Yuzbasioglu E, Rasier R, et al. Intravitreal ranibizumab in the treatment of cystoid macular edema associated with retinitis pigmentosa. J Ocul Pharmacol Ther. 2009;25(6):545-550.

16. Garcia-Arumi J, Martinez V, Sararols L, Corcostegui B. Vitreoretinal surgery for cystoid macular edema associated with retinitis pigmentosa. Ophthalmology. 2003;110(6):1164-1169.
Clinical Ophthalmology

\section{Publish your work in this journal}

Clinical Ophthalmology is an international, peer-reviewed journal covering all subspecialties within ophthalmology. Key topics include: Optometry; Visual science; Pharmacology and drug therapy in eye diseases; Basic Sciences; Primary and Secondary eye care; Patient Safety and Quality of Care Improvements. This journal is indexed on PubMed

Submit your manuscript here: http://www.dovepress.com/clinical-ophthalmology-journal

\section{Dovepress}

Central and CAS, and is the official journal of The Society of Clinical Ophthalmology (SCO). The manuscript management system is completely online and includes a very quick and fair peer-review system, which is all easy to use. Visit http://www.dovepress.com/testimonials. php to read real quotes from published authors. 